\title{
A population-based retrospective cohort study analyzing contemporary trends in the surgical management of urinary stone disease in adults
}

Dor Golomb $^{1}$; Sumit Dave ${ }^{1}$; Fernanda Gabrigna Berto ${ }^{1}$; J. Andrew McClure ${ }^{2}$; Blayne Welk ${ }^{1}$; Peter Wang ${ }^{1}$; Jennifer Bjazevic ${ }^{1}$; Hassan Razvi ${ }^{1}$

${ }^{1}$ Division of Urology, Schulich School of Medicine and Dentistry, Western University, London, ON, Canada; ${ }^{2}$ Department of Surgery, Schulich School of Medicine and Dentistry London Health Sciences Centre, London, ON, Canada

Acknowledgements: This study was supported by ICES, which is funded by an annual grant from the Ontario Ministry of Health (MOH) and the Ministry of Long-Term Care (MLTC). Parts of this material are based on data and/or information compiled and provided by CIHI. However, the analyses, conclusions, opinions and statements expressed in the material are those of the authors, and not necessarily those of CIHI. The analyses, conclusions, opinions, and statements expressed herein are solely those of the authors and do not reflect those of the funding or data sources; no endorsement is intended or should be inferred.

Cite as: Golomb D, Dave S, Berto FG, et al. A population-based retrospective cohort study analyzing contemporary trends in the surgical management of urinary stone disease in adults. Can Urol Assoc J 2021 November 18; Epub ahead of print. http://dx.doi.org/10.5489/cuaj.7474

Published online November 18, 2021

Corresponding author: Dr. Hassan Razvi, Division of Urology, Schulich School of Medicine and Dentistry, Western University, London, ON, Canada; hrazvi@uwo.ca

$* * *$

\section{Abstract}

Introduction: We aimed to review the trends and incidence of surgical intervention for adults with upper urinary tract stones in Ontario, Canada, and to hypothesize potential causes for the observed changes.

Methods: We carried out a retrospective, population-based cohort study using administrative databases held at the Institute of Clinical Evaluative Sciences (IC/ES) to identify all adults ( $\geq 18$ years) who underwent surgical treatment for urolithiasis, defined by records using a combination of both hospital and physician billing from 2002-2019. Descriptive statistics were used to summarize baseline patient demographics, and surgical trends were analyzed using the Cochrane-Armitage test for trend.

Results: From 2002-2019, 140263 patients were treated surgically for urolithiasis. During this time period, the total number of surgically treated stone disease increased by $80.5 \%$. By type of 
procedure, percutaneous nephrolithotomy (PCNL) increased by $187 \%$ and ureteroscopy (URS) increased by $158 \%$, while the number of shockwave lithotripsy (SWL) declined by $31.4 \%$. The adult population in Ontario in the years evaluated grew by $24.4 \%$. The number of surgical procedures per 100000 people over this time grew by $45.3 \%$. For every $1 \%$ increase in the population, there was a $2.6 \%$ rise in stone-related surgical procedures.

Conclusions: The number of stone-related surgical procedures performed rose significantly and cannot be accounted for by population growth alone. This rise was proportionally larger in the female population, further supporting a narrowing of the gender gap in urinary stone disease. The reasons for the increase are likely multifactorial and may imply an increasing incidence of surgically treated stone disease. The change in the proportion of URS and SWL performed may demonstrate a continued shift in surgical preference or may be reflective of resource limitations and availability. The increase in PCNL volumes may also suggest a greater complexity of cases. These findings should be considered for future resource planning and require further study.

\section{Introduction}

Urolithiasis is a prevalent disease, affecting $10 \%$ of the population worldwide ${ }^{1}$. The prevalence and incidence of urinary stones has increased over the last three decades ${ }^{2,3}$. Technological refinements have resulted in miniaturization of the endoscopes used in these procedures. With these advancements, URS and laser lithotripsy has become increasingly popular.

A study looking into surgical trends between 1990-1998 reported that the number of PCNL's remained stable, URS's increased by 53\%, while the number of SWL's, decreased by $15 \%{ }^{4}$. Ordon et al. ${ }^{5}$, reported that between the years 1991-2010, in Ontario, Canada, the utilization of URS increased significantly from $25 \%$ to $59 \%$ of all procedures, whereas PCNL remained unchanged in its' utilization and SWL decreased significantly from $69 \%$ to $34 \%$ of all procedures. We felt it was important to revisit the issue using a more contemporary cohort, and to explore possible reasons for any trends that might be observed.

In this study we reviewed the trends in surgical intervention for urolithiasis of the upper urinary tract in patients living in the province of Ontario, Canada. The aims of our study were to determine if the total number of surgical procedures was increasing relatively to the general population. We were also interested in determining if the previously noted surgical modality trends were persistent and hypothesize potential epidemiological reasons for the observed changes.

\section{Methods}

This retrospective population-based cohort study included all adult patients ( $\geq 18$ years of age) who underwent a minimally invasive surgical treatment for urolithiasis (calculi located within the kidney or ureter) in the province of Ontario, Canada, from April 1 ${ }^{\text {st }}, 2002$ to March $31^{\text {st }}$, 
2019. Although urinary stone disease has a high recurrence rate, we chose to exclude patients with prior surgical procedures in the last 10 years and aimed to capture those who had their initial presentation during the study period and exclude patients who underwent secondary procedures for residual fragments. We excluded non-Ontario residents, patients with missing or invalid age, sex, or date of death information, open cases and those who had undergone previous surgical management of urolithiasis within the last 10-years. Figure S1 in Appendix describes the cohort selection process. Hospital and same day surgery services are recorded in the Canadian Institute for Health Information's (CIHI) Discharge Abstract Database and Same Day Surgery databases, whereas physician billing records are maintained in the Ontario Health Insurance Plan (OHIP) database. The Registered Persons Database was also used to obtain baseline patient characteristics. These datasets were linked using unique encoded identifiers (Table S1) and analyzed at ICES Western. Reporting of this study follows the RECORD statement ${ }^{6}$ (Table S2).

\section{Variable definitions}

Surgical management of urolithiasis was identified using inclusion in either the OHIP or CIHI databases. Duplicate records were reconciled by keeping one record and only the first procedure per patient was included in the analysis. Since this is primarily a trends study, a combination of either/or OHIP and CIHI codes were used as inclusion criteria to ensure we captured the largest possible population. The CIHI codes were more definitive in determining the index modality and were used as the primary inclusion code. The OHIP codes utilized were a combination of Scodes with either simultaneous $\mathrm{E}$ codes or independent $\mathrm{Z}$ codes (Table S1). A large degree of agreement was noted between the two databases. Eligible patients were categorized into three groups based on their index surgical modality: URS, SWL and PCNL. Patients who underwent multiple surgical treatment modalities on the same date were assigned to a group according to the following hierarchy: PCNL, SWL, and URS. This hierarchy was based on the fact that if PCNL and SWL was performed, it is likely that URS was probably a secondary surgical modality. Patient baseline characteristics captured included: age, sex, rural/urban residence (population above/under 10,000), calculus location (ureter or kidney), and first imaging modality used for assessment of stone disease in the 90 days before the surgical procedure. If both $\mathrm{CT}$ and US occurred on the same date, the first imaging modality was categorized as CT.

\section{Statistical analysis}

Data is reported as means and standard deviations. The linear trend in the overall number of cases per year was assessed using a linear regression model. Trends across the study period in terms of patient sex and surgical intervention were evaluated using the Cochrane-Armitage test for trend. For all analyses, reported p-values are from 2-tailed tests where a value of $<0.05$ was considered statistically significant. The main assumptions of this model include that the dependent variable and errors of the linear regression model are normally distributed. All analyses were performed using SAS EG version 7.15 (SAS Institute, Cary, NC, USA). 


\section{Results}

Over the 17-year study period, 140,263 adult patients underwent a minimally invasive surgical procedure for management of urolithiasis in Ontario. The mean age of patients undergoing surgical management for urolithiasis was 54 years of age and $60.5 \%$ of patients were male. Detailed demographic and preoperative data are given in Table 1.

CT scans were predominantly used as the first imaging modality (66\%), compared to US scans which were only used as the first imaging modality in $29 \%$ of the cases overall. Throughout the study period the number of patients undergoing CT scans preoperatively and the proportion of patients undergoing CTs scan as their first imaging modality increased significantly ( $\mathrm{p}<0.0001$ ). Specifically, for PCNL, the first imaging modality used was CT for $44.2 \%$ of patients, US for $22.7 \%$ and $2.6 \%$ had both modalities on the same day. An additional $14 \%$ of patients underwent CT imaging after their first imaging investigation so that overall, $58.2 \%$ underwent $\mathrm{CT}$ at some time preoperatively.

The proportion of females undergoing surgery for urolithiasis increased from $39.5 \%$ in 2002 to $42 \%$ in 2018 ( $p<0.0001$ ). This corresponded to a percentage increase of $112 \%$ in females undergoing surgical management for stone disease, compared to an increase of only $60 \%$ in the male population (Figure 1). The overall increase in the population of females and males during that same time period was $22.5 \%$ and $23.8 \%$ respectively.

During the studied period, the adult population in Ontario grew by $24.4 \%$, from $9,294,774$ to $11,564,360$. The total number of surgical procedures presented a steady increase, rising by $80.4 \%$, from 5,946 cases in 2002 to 10,732 cases in 2018 , resulting in an average yearly rise of 3.7\%. In 2002, the annual stone related surgical intervention rate in Ontario was 64 per 100,000 population while in 2018 it was 93 per 100,000 population, a 45.3\% rise (Figure 2). When considering the adult population in Ontario relative to the number of stone related surgical procedures, it was observed that for every $1 \%$ increase in the population, there was a $2.6 \%$ increment in stone related surgical procedures.

The number of URS and PCNL procedures per 100,000 population grew from 29.6 to 61.5 and 6.97 up to 16.1 respectively. The number of SWL procedures per 100,000 population decreased from 27.4 in 2002, down to 15.1 in 2018 (Figure 3). There was a steady yearly increase in the number of PCNLs performed, from 648 to 1,864 cases, corresponding to a 7\% annual increase and a $185 \%$ rise over the study duration. URS increased at an average annual rate of $6 \%$, resulting in a total increase of $158 \%$ over the study period, from 2,751 cases in 2002 to 7,121 cases in 2018 . Conversely, SWL procedures were observed to steadily decline, with a yearly decrease of $2 \%$, an overall decrease of $31.4 \%$, from 2,547 down to 1,747 procedures (Figure 3). Linear regression analysis demonstrated a significant increase in both URS and PCNL and a decrease in SWL over the study period ( $<<0.005$ overall for all 3 modalities). 


\section{Discussion}

The incidence and prevalence of urolithiasis has increased over the years. This rise in prevalence has been reported in studies from Europe ${ }^{7}$, North America ${ }^{8}$ and Asia ${ }^{9}$. Increases in the number of patients diagnosed with stones may subsequently result in a rise in the number of stone-related surgical procedures. Previous studies reported on the rising number of stone related surgical procedures over the years ${ }^{10,11}$. Ordon et al. ${ }^{5}$ reported that the number of URS increased significantly, while the number of PCNL procedures remained unchanged and the number of SWL decreased from $69 \%$ to $34 \%$ of all procedures. We report on a continued increase in the number of URSs performed, but in contrary to Ordons' report, we noted a rise in the number of PCNLs. Similar to their study, we noted a continued decrease in the utilization of SWL. The rise in number of PCNLs performed may be indicative of a greater stone complexity as well as a growing number of practicing urologists in Ontario trained in PCNL. Although we could not calculate stone disease incidence or prevalence figures with the dataset we used, we observed a steady rise in stone related surgery, with a total increase of $80.5 \%$ in the 12 years assessed. We also report on a significant percentage increase in females undergoing stone related procedures compared to the male population (Figure 1).

The reasons for the observed increase in the surgical intervention of stone disease and the change in surgical modalities are likely multifactorial and are most probably not just strictly related to the increase in the number of patients afflicted with stones observed in recent worldwide trends ${ }^{7,8,9}$. In the remainder of this report, we explore the possible explanations for the increase in interventions.

\section{Population size and age}

It is also well-known that the prevalence of urolithiasis increases with age ${ }^{8}$. In Ontario, the adult population aged 18 years and above, increased by $24.1 \%$, in the years assessed. Moreover, the age group of 40-69 years old increased significantly by $29 \%$, from $4,336,116$ up to $5,597,172^{8}$ (Figure 4). The surgical intervention rate in the adult population in Ontario in the year 2002 was 64 per 100,000 population, rising to 93 in 2018. The number of stone related surgical procedures per 100,000 people grew in those years by $45.3 \%$ (Figure 3 ). In our data we noted that for every increase by $1 \%$ in the population size, there was a $2.6 \%$ rise in stone related surgical procedures. We were surprised to find that as the population rose by $24.4 \%$ in the years investigated, the number of stone related procedures outpaced the population, and had risen by $80.5 \%$. By extrapolating the yearly average increase in population with the yearly increase in the number of surgical procedures, we predict that if the population continues to grow at the same rate and the rate of stone disease remains constant, the total number of stone related surgeries will increase to almost 14,000 by the year 2025 . We hypothesize that both the increase in the overall adult population and specifically the age group of 40-69 years-old, may be correlated with the increased number of surgical procedures. If our prediction is correct, this will have a significant impact on patient care and resource management. 


\section{Obesity and diet}

There are two main dietary factors that may play a major role in the upsurge in the prevalence of urolithiasis: quantity and quality of food. Studies have shown a direct correlation between the prevalence of urolithiasis and an elevated body mass index (BMI) ${ }^{12-15}$. The prevalence of obesity in Ontario has increased significantly over the past three decades ${ }^{16}$, growing by $31 \%$ between 2004-2015. According to a 2015 Canadian Community Health Survey, $32 \%(3,444,100)$ of Canadians were considered overweight (BMI 25-29.9) and 26\% $(2,768,100)$ were found to be obese $(\mathrm{BMI} \geq 30)^{17}$ (Figure 5). The percentage increase in obesity was $21.3 \%$ and $45 \%$ in females and males respectively. Unfortunately, we were unable to capture BMI data for our study cohort; however, the rising rates of obesity within the province of Ontario have likely contributed to the increase in surgically managed urinary stone disease observed.

The quality of the diet, mainly low urinary volumes due to insufficient fluid intake, combined with a high sodium and protein consumption, may also play a role in stone formation $^{18}$. A recent study by the Canadian government reported that Canadians consume a staggering amount of 3,400 mg of sodium daily ${ }^{19}$. We hypothesize therefore that both the quality and quantity of the diet consumed may have contributed to the rising numbers of urolithiasis related surgical procedures.

\section{Number of practicing urologists}

According to the Canadian Urological Association (CUA), the number of practicing urologists in Ontario has risen by $70.6 \%$, from 200 in 2002 up to 283 in $2018^{20}$. Moreover, the number of fellowship trained endourologists also increased. Welk et al. ${ }^{21}$ reported that among urology graduates, 39\% proceeded to fellowship in minimally invasive urology/endourology. Presumably, these urologists feel more comfortable performing PCNLs and URSs.

\section{Improved diagnostics}

According to the Canadian Medical Imaging Inventory, in 2017 there were 561 CT units in Canada, up from 419 in 2007. It was estimated that a total of 5.61 million CT examinations were performed in 2017, up from 3.38 million in $2007^{22}$. With improved diagnostics and shorter waiting time, the number of patients diagnosed with renal stones would probably rise, leading to more patients seeking medical attention and treatment. This was also reflected in our data, where we observed a significant increase in the number of patients undergoing preoperative CT scans and having a CT scan as their initial imaging modality.

\section{Decreasing number of $S W L$ procedures}

Our study has demonstrated that the number of SWL procedures being performed in Ontario has decreased during the years of our evaluation. We believe that the cause for this is multifactorial. Most importantly, there are only three shock wave lithotripsy units in Ontario, which results in limited access to this technology. $11.3 \%$ of patients in our cohort live in a rural area, which may have led them to travel a great distance in order to undergo treatment. This may have resulted in 
patients preferring a different treatment modality, such as URS, performed in a local community medical center. This is reflected in our data as a smaller proportion of patients living in rural areas underwent SWL (8.3\%) compared with either URS (12.6\%) or PCNL (11.7\%). Additionally, the reported lower stone free rate of SWL compared to URS ${ }^{23}$ may lead urologists and patients to choose one modality over another. This data should be taken into consideration for future resource planning regarding the surgical management of urolithiasis.

In our study we were able to access a large contemporary patient cohort treated in a universal care, single-payer health care system over a long study period. There are several limitations to our study include the retrospective study design with all its inherent biases. The administrative databases we utilized were unable to provide certain clinical data such as patient dietary habits, stone composition and metabolic evaluation results. Unfortunately, we did not capture the rates of other imaging modalities, like X-ray KUB, as one of the goals of this study was to capture the trends in CT imaging usage versus Ultrasound for stone treatment. We are therefore, unable to determine the overall rate of preoperative imaging in our study cohort. We acknowledge an additional limitation, only capturing imaging completed 90 -days before surgery, which may have resulted in missing some imaging that took place earlier than that. This is a trends study and therefore aimed to capture all patients who underwent a surgical procedure for urolithiasis. Therefore, when including both OHIP and CIHI coding data we acknowledge that there is a potential for misclassification of these patients according to their primary surgical modality (SWL, URS, PCNL). We attempted to minimize this with using the CCI codes as the primary source to classify the stone treatment modality. Our study only included the first surgical procedure captured during the study period; therefore, patients who underwent multiple procedures for treatment of the same stone or residual fragments, as well as patients with recurrent stones were excluded from the analysis. Consequently, our data will not reflect the total number of surgical stone procedures in the Ontario population and overall urinary stone disease incidence and prevalence data cannot be extracted from this cohort.

\section{Conclusions}

The number of surgical procedures performed on patients with upper tract urinary stones in Ontario, Canada has significantly risen during the years assessed. Endoscopic procedures have increased while the utilization of SWL has decreased. The overall increase in surgical intervention is most likely multi-factorial. There was a higher percentage increase in females undergoing stone related surgical procedures that was independent of changes in population growth and increasing BMI levels, which further supports the narrowing of the gender gap in urinary stone disease. We believe that health care providers should be aware of the upward trend in the number of stone-related surgical procedures and plan appropriately with funding, personnel and equipment. Further study is required to determine which factors are driving the increasing number of surgical procedures and if they reflect a true increase in stone incidence. We have hypothesized a number of potential causes for the suspected increase in incidence and 
additional work is required to better understand which parameters are most important and to determine if any are modifiable. 


\section{References}

1. Curtin J and Sampson M: Greenhouse effect and renal calculi. Lancet (London, England) 1989; 2: 1110.

2. Turney BW, Reynard JM, Noble JG, et al: Trends in urological stone disease. BJU Int. 2012; 109: 1082-1087.

3. Romero V, Akpinar $\mathrm{H}$ and Assimos DG: Kidney stones: a global picture of prevalence, incidence, and associated risk factors. Rev. Urol. 2010; 12: e86-96.

4. Kerbl K, Rehman J, Landman J, et al: Current management of urolithiasis: Progress or regress? In: Journal of Endourology.Vol 16. Mary Ann Liebert Inc. 2002; pp 281-288.

5. Ordon M, Urbach D, Mamdani M, et al: The surgical management of kidney stone disease: A population based time series analysis. J. Urol. 2014; 192: 1450-1456.

6. Benchimol EI, Smeeth L, Guttmann A, et al: The REporting of studies Conducted using Observational Routinely-collected health Data (RECORD) Statement. PLoS Med. 2015; 12.

7. Indridason OS, Birgisson S, Edvardsson VO, et al: Epidemiology of kidney stones in Iceland: A population-based study. Scand. J. Urol. Nephrol. 2006; 40: 215-220.

8. Scales CD, Smith AC, Hanley JM, et al: Prevalence of kidney stones in the United States. Eur. Urol. 2012; 62: 160-165.

9. Iguchi M, Umekawa T, Katoh Y, et al: Prevalence of urolithiasis in Kaizuka City, Japan An epidemiologic study of urinary stones. Int. J. Urol. 1996; 3: 175-179.

10. Chung KJ, Kim JH, Min GE, et al: Changing Trends in the Treatment of Nephrolithiasis in the Real World.

11. Patel NH, Parikh SS, Bloom JB, et al: Contemporary trends in percutaneous nephrolithomy across New York State: A review of the statewide planning and research cooperative system. J. Endourol. 2019; 33: 699-703.

12. Taylor EN, Stampfer MJ and Curhan GC: Obesity, weight gain, and the risk of kidney stones. J. Am. Med. Assoc. 2005; 293: 455-462.

13. Siener R, Glatz S, Nicolay C, et al: The role of overweight and obesity in calcium oxalate stone formation. Obes. Res. 2004; 12: 106-113.

14. Ekeruo WO, Tan YH, Young MD, et al: Metabolic risk factors and the impact of medical therapy on the management of nephrolithiasis in obese patients. In: Journal of Urology.Vol 172. Lippincott Williams and Wilkins 2004; pp 159-163.

15. Najeeb Q, Masood I, Bhaskar N, et al: Effect of BMI and urinary $\mathrm{pH}$ on urolithiasis and its composition. Saudi J. Kidney Dis. Transpl. 2013; 24: 60-66.

16. Anon: Measured adult body mass index (BMI) in Ontario in the years 2004 and 2015. Canadian Community Health Survey. Available at: https://www150.statcan.gc.ca/t1/tbl1/en/tv.action?pid=1310079401\&pickMembers\%5B0 $\% 5 \mathrm{D}=1.7 \&$ pickMembers $\% 5 \mathrm{~B} 1 \% 5 \mathrm{D}=2.1$ \&pickMembers $\% 5 \mathrm{~B} 2 \% 5 \mathrm{D}=3.1 \&$ pickMembers $\% 5 \mathrm{~B} 3 \% 5 \mathrm{D}=5.1 \&$ cubeTimeFrame.startYear $=2004 \&$ cubeTimeFrame.endYear=2015\&refe rencePeriods $=20040101 \% 2 \mathrm{C} 20150101$, accessed October 2, 2020.

17. Anon: Measured adult body mass index (BMI) (World Health Organization classification), by age group and sex, Canada and provinces, Canadian Community Health Survey - Nutrition. Available at: 
https://www150.statcan.gc.ca/t1/tb11/en/tv.action?pid=1310079401, accessed August 26, 2020.

18. Turney BW, Appleby PN, Reynard JM, et al: Diet and risk of kidney stones in the Oxford cohort of the European Prospective Investigation into Cancer and Nutrition (EPIC). Eur. J. Epidemiol. 2014; 29: 363-369.

19. Anon: Sodium in Canada - Canada.ca. Available at: https://www.canada.ca/en/healthcanada/services/food-nutrition/healthy-eating/sodium.html, accessed August 25, 2020.

20. Anon: Information provided by "CUA Membership Services."

21. Welk B, Kodama R and MacNeily A: The newly graduated canadian urologist: Overtrained and underemployed? J. Can. Urol. Assoc. 2013; 7: 10-15.

22. Sinclair A, Morrison A, Young C, et al: CADTH OPTIMAL USE REPORT The Canadian Medical Imaging Inventory, 20172.

23. Fankhauser CD, Hermanns T, Lieger L, et al: Extracorporeal shock wave lithotripsy versus flexible ureterorenoscopy in the treatment of untreated renal calculi. Clin. Kidney J. 2018; 11: 364-369.

24. Statistics Canada: Population estimates in Ontario by age and sex between 2002-2018. Available at: https://www150.statcan.gc.ca/t1/tbl1/en/tv.action?pid=1710000501\&pickMembers\%5B0 $\% 5 \mathrm{D}=1.7 \&$ pickMembers\%5B1\%5D $=2.1 \&$ cubeTimeFrame.startYear=2002\&cubeTimeFr ame. endYear $=2018 \&$ referencePeriods $=20020101 \% 2$ C20180101, accessed October 2, 2020.

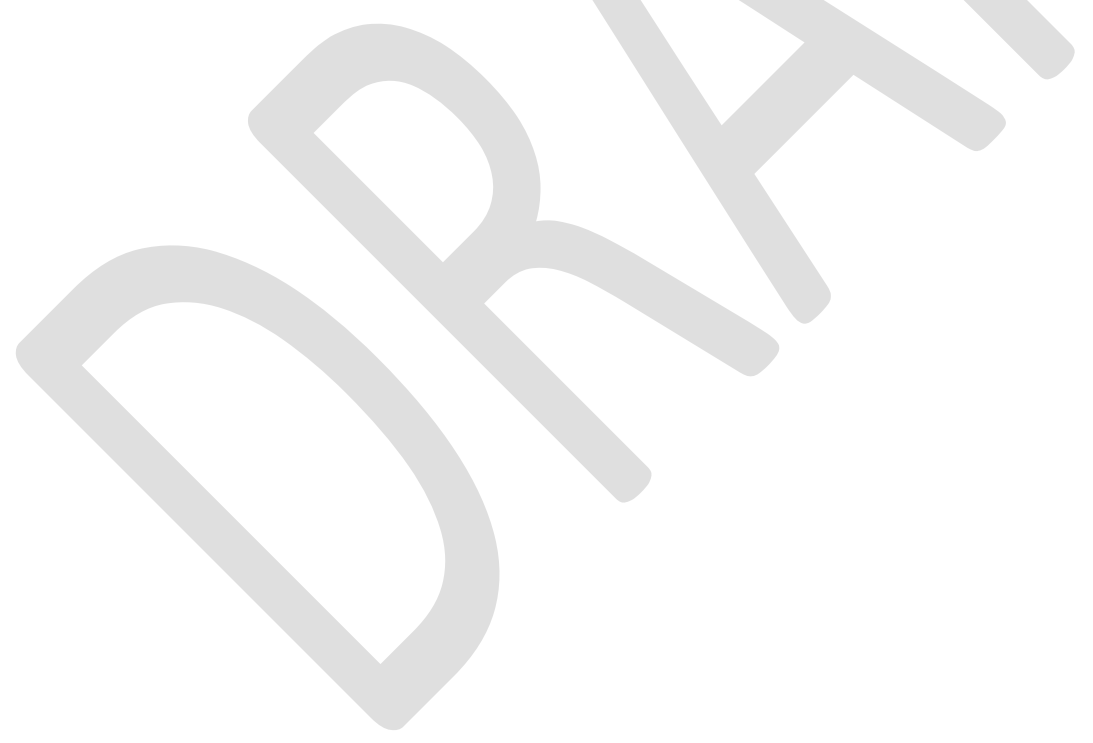




\section{Figures and Tables}

Fig. 1. Percentage increase in surgical intervention in males and females from 2002-2019.

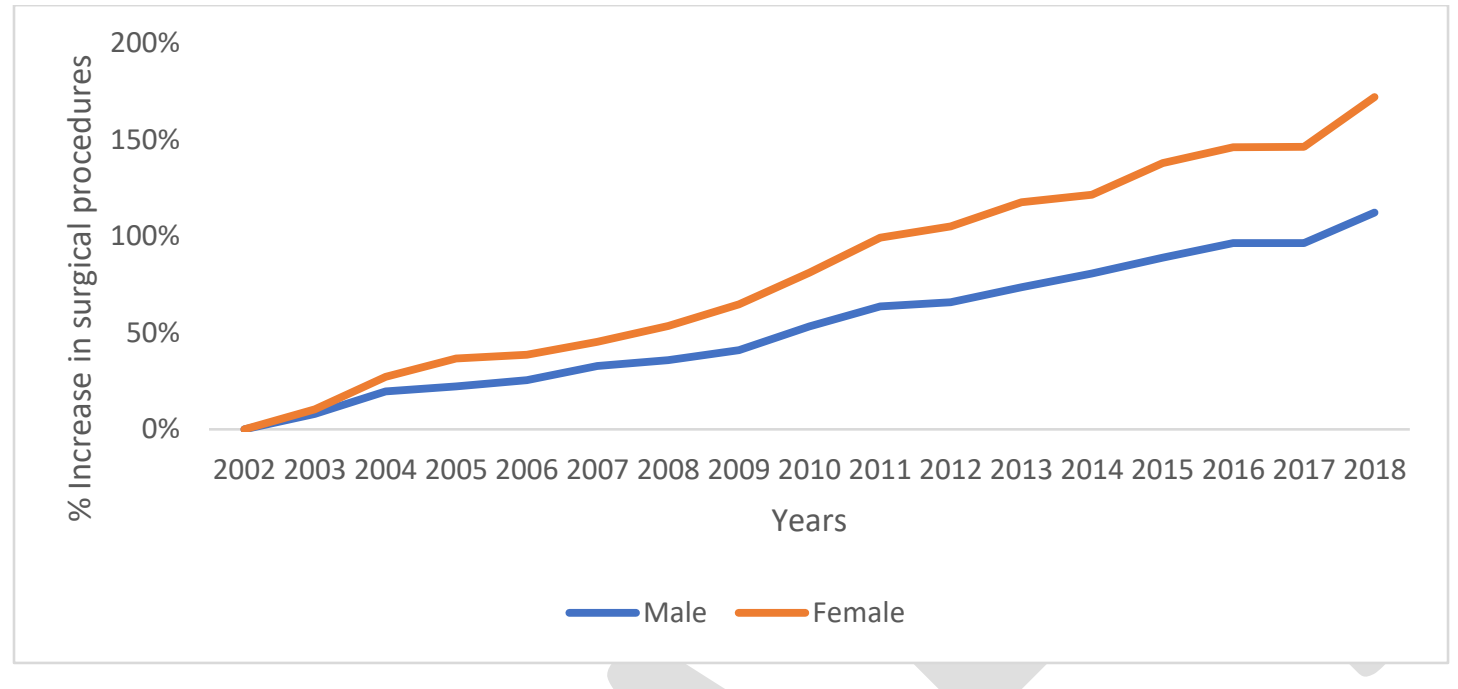

Fig. 2. Adult population in Ontario and number of stones related surgical procedures from 20022019.

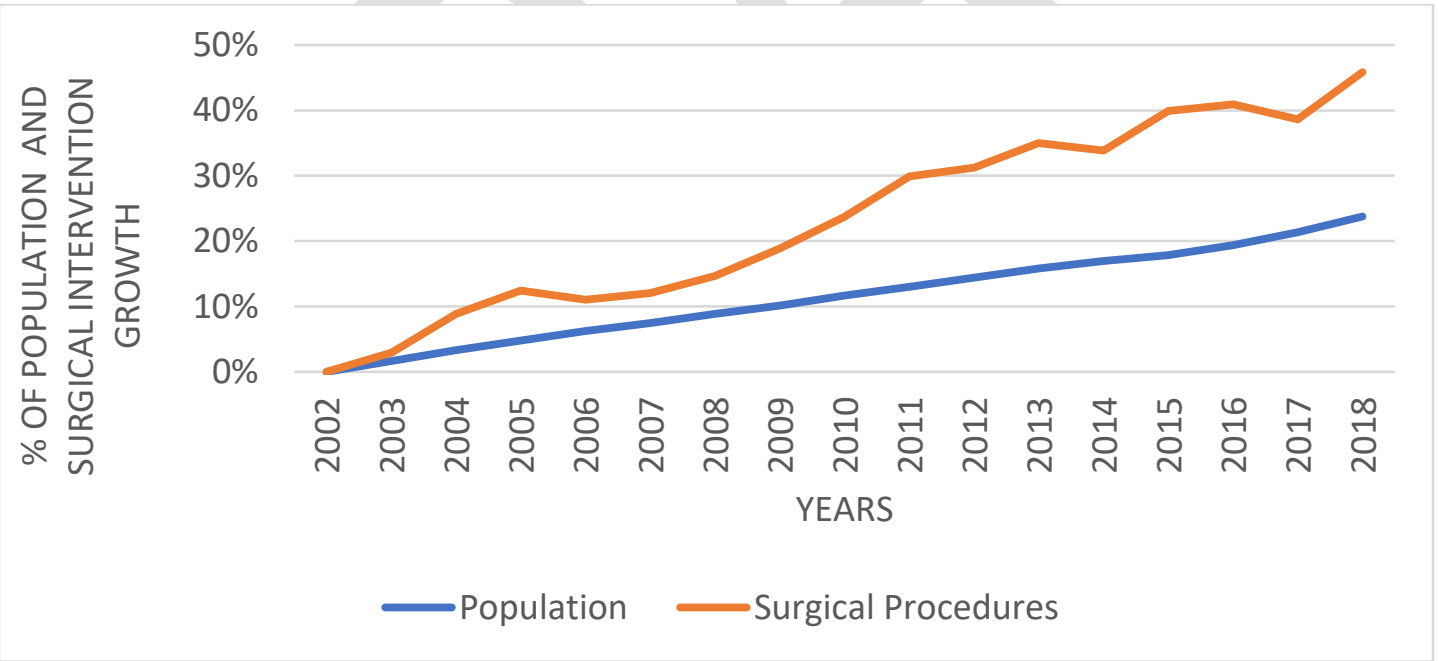


Fig. 3. Surgical intervention rate with percutaneous nephrolithotomy (PCNL), ureteroscopy (URS), and shockwave lithotripsy (SWL) from 2002-2019.

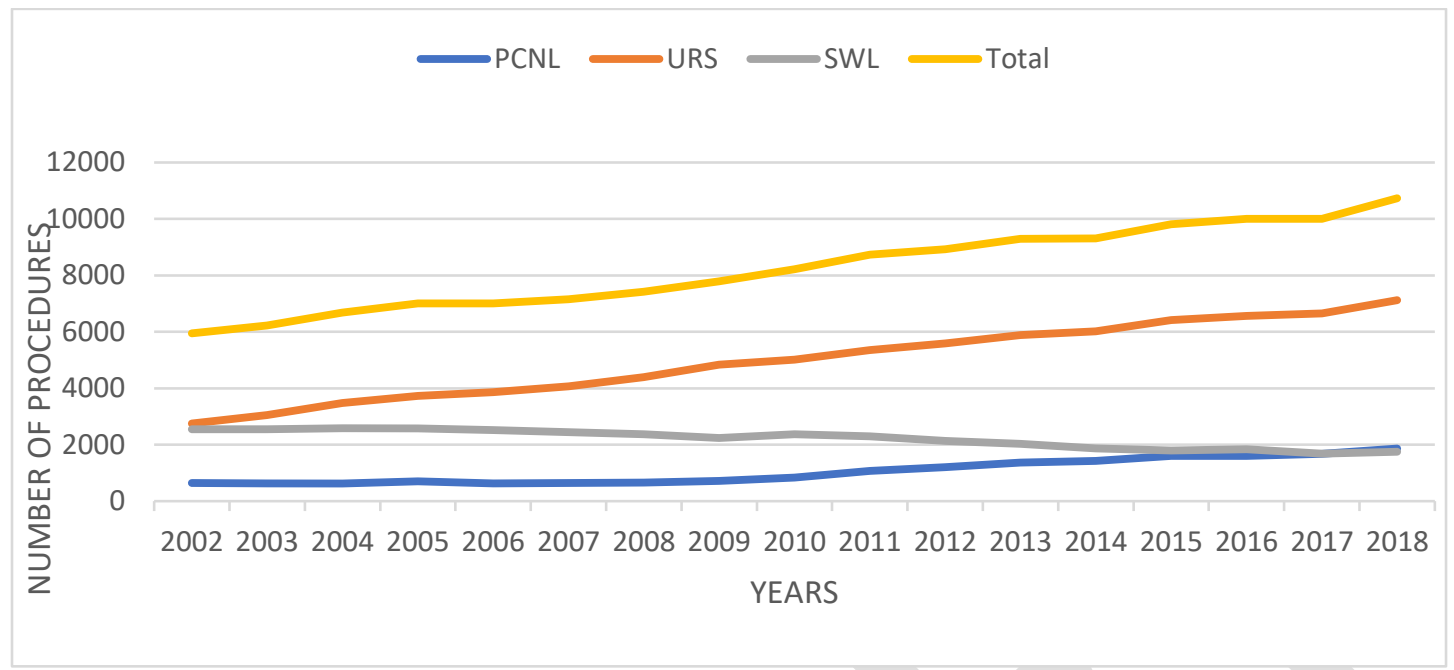

Fig. 4. Adult population in Ontario stratified by age from $2002-2019 .^{24}$

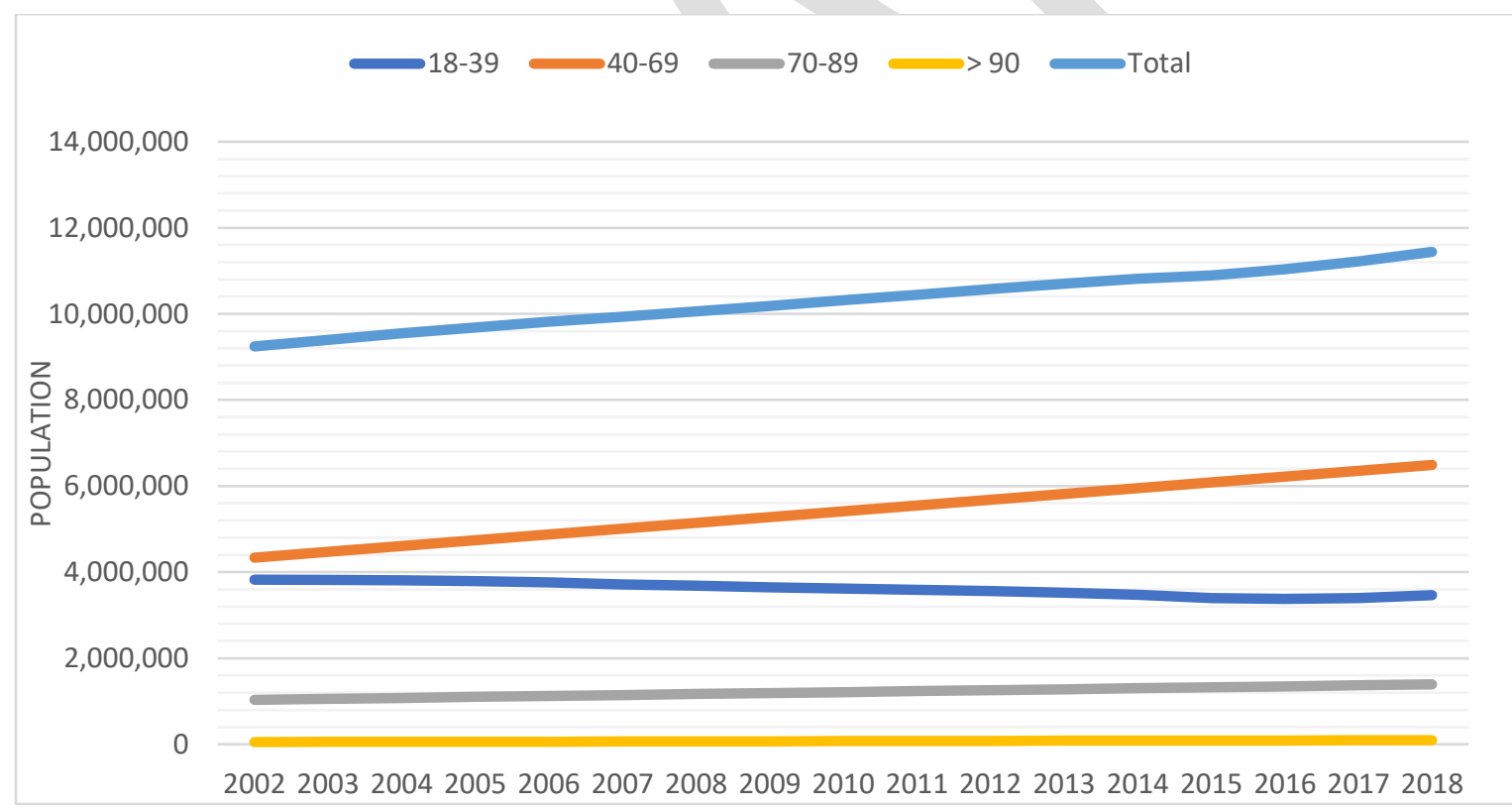


Fig. 5. Measured adult body mass index (BMI) in Ontario in 2004 and $2015 .{ }^{16}$

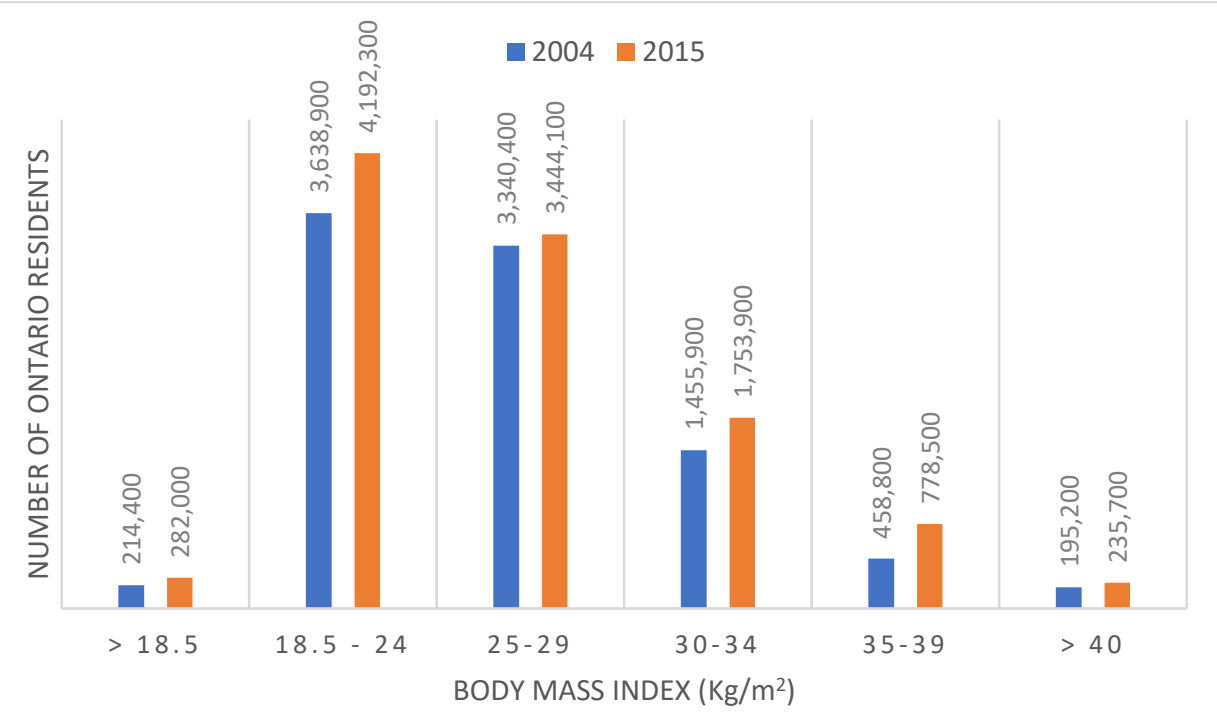
Table 1. Demographics of patients undergoing surgical treatment for urolithiasis in
Ontario from 2002-2019

\begin{tabular}{|c|c|c|c|}
\hline & $\begin{array}{c}\text { PCNL } \\
(\mathrm{n}=17 \text { 892) }\end{array}$ & $\begin{array}{c}\text { URS } \\
(\mathrm{n}=87784)\end{array}$ & $\begin{array}{c}\text { SWL } \\
(\mathrm{n}=37 \text { 587) }\end{array}$ \\
\hline Age $($ mean \pm SD $)$ & $56.09 \pm 15.05$ & $54.35 \pm 15.76$ & $52.9 \pm 14.27$ \\
\hline Males, n (\%) & $10029(56.1)$ & $50813(59.9)$ & $23771(63.2)$ \\
\hline Rural residence, n (\%) & $\begin{array}{l}2082 \\
(11.6)\end{array}$ & $\begin{array}{l}10736 \\
(12.7)\end{array}$ & $\begin{array}{l}3085 \\
(8.2)\end{array}$ \\
\hline $\begin{array}{l}\text { First imaging modality used, } \mathrm{n}(\%) \\
\text { CT } \\
\text { US }\end{array}$ & $\begin{array}{l}7917(44) \\
4066(23)\end{array}$ & $\begin{array}{l}48809(58) \\
20983(25)\end{array}$ & $\begin{array}{c}17860(48) \\
8337(22)\end{array}$ \\
\hline Preoperative CT scan obtained, n (\%) & $10462(58)$ & $64153(75)$ & $22144(59)$ \\
\hline $\begin{array}{l}\text { Stone location, n (\%) } \\
\text { Ureter } \\
\text { Kidney } \\
\text { Missing data }\end{array}$ & $\begin{array}{c}3774(21) \\
12814(72) \\
1304(7)\end{array}$ & $\begin{array}{c}53541(63) \\
23308(28) \\
10935(9)\end{array}$ & $\begin{array}{l}7477(20) \\
17043(45) \\
13067(35)\end{array}$ \\
\hline
\end{tabular}

CT: computed tomography; PCNL: percutaneous nephrolithotripsy; SD: standard deviation; SWL: shockwave lithotripsy; URS: ureteroscopy; US: ultrasound. 